\title{
BMJ
}

\section{Effect of a multimodal high intensity exercise intervention in cancer patients undergoing chemotherapy: randomised controlled trial}

\author{
Lis Adamsen, professor clinical nursing, ${ }^{1,2}$ Morten Quist, research physiotherapist , ${ }^{2}$ Christina Andersen, \\ research nurse , ${ }^{2}$ Tom Møller, research nurse , ${ }^{2}$ Jørn Herrstedt, professor clinical oncology , ${ }^{3}$ Dorte Kronborg, \\ associate professor, ${ }^{4}$ Marie T Baadsgaard, research assistant, ${ }^{2}$ Kirsten Vistisen, MD , ${ }^{5}$ Julie Midtgaard, \\ research psychologist , ${ }^{2}$ Birgitte Christiansen, staff study nurse , ${ }^{5}$ Maria Stage, student assistant, ${ }^{2}$ Morten T \\ Kronborg, BSc Stud Act, ${ }^{6}$ Mikael Rørth, professor ${ }^{7}$
}

\section{Institute of Public Health University of Copenhagen, Copenhagen, Denmark}

${ }^{2}$ University Hospitals Centre for Nursing and Care Research,

Copenhagen University Hospital, DK-2100 Copenhagen, Denmark

${ }^{3}$ Department of Oncology, Odense University Hospital, Odense,

Denmark

${ }^{4}$ Centre for Statistics, Copenhagen Business School, Frederiksberg, Denmark

${ }^{5}$ Department of Oncology, Herlev University Hospital, Herlev,

Denmark

${ }^{6}$ Department of Mathematical

Sciences, University of

Copenhagen, Copenhagen,

Denmark

${ }^{7}$ Department of Oncology,

Copenhagen University Hospital

Correspondence to: L Adamsen,

University Hospitals Centre for

Nursing and Care Research,

Copenhagen University Hospital,

DK-2100 Copenhagen, Denmark

la@ucsf.dk

Cite this as: $B M J$ 2009;339:b3410 doi:10.1136/bmi.b3410 advanced disease.

\section{ABSTRACT}

Objective To assess the effect of a multimodal group exercise intervention, as an adjunct to conventional care, on fatigue, physical capacity, general wellbeing, physical activity, and quality of life in patients with cancer who were undergoing adjuvant chemotherapy or treatment for

Design Randomised controlled trial.

Setting Two university hospitals in Copenhagen, Denmark.

Participants 269 patients with cancer; 73 men, 196 women, mean age 47 years (range 20-65) representing 21 diagnoses. Main exclusion criteria were brain or bone metastases. 235 patients completed follow-up. Intervention Supervised exercise comprising high intensity cardiovascular and resistance training, relaxation and body awareness training, massage, nine hours weekly for six weeks in addition to conventional care, compared with conventional care.

Main outcome measures European Organization for Research and Treatment of Cancer Quality of Life Questionnaire (EORTC QLQ-C30), Medical Outcomes Study Short Form (MOS SF-36), Leisure Time Physical Activity Questionnaire, muscular strength (one repetition maximum), maximum oxygen consumption $\left(\mathrm{Vo}_{2}\right.$ max). Statistical methods The general linear model was used for continuous outcome while analysis of associates between categorical outcomes was performed as analysis of marginal homogeneity in contingency tables.

Results Adjusted for baseline score, disease, and demographic covariates, the intervention group showed an estimated improvement at six weeks for the primary outcome, fatigue, of -6.6 points ( $95 \%$ confidence interval -12.3 to $-0.9, \mathrm{P}=0.02$; effect size $=0.33,0.04$ to 0.61 ). Significant effects were seen on vitality (effect size 0.55 , $95 \% \mathrm{Cl} 0.27$ to 0.82$)$, physical functioning $(0.37,0.09$ to $0.65)$, role physical $(0.37,0.10$ to 0.64$)$, role emotional $(0.32,0.05$ to 0.59$)$, and mental health $(0.28,0.02$ to $0.56)$ scores. Improvement was noted in physical capacity: estimated mean difference between groups for maximum oxygen consumption was $0.16 \mathrm{l} / \mathrm{min}(95 \% \mathrm{Cl}$ 0.1 to $0.2, \mathrm{P}<0.0001$ ) and for muscular strength (leg press) was $29.7 \mathrm{~kg}$ (23.4 to 34.9, P<0.0001). No significant effect was seen on global health status/quality of life.

Conclusion A supervised multimodal exercise intervention including high and low intensity components was feasible and could safely be used in patients with various cancers who were receiving adjuvant chemotherapy or treatment for advanced disease. The intervention reduced fatigue and improved vitality, aerobic capacity, muscular strength, and physical and functional activity, and emotional wellbeing, but not quality of life.

Trial registration Current Controlled trials ISRCTN05322922.

\section{INTRODUCTION}

An increasing number of patients with cancer are offered chemotherapy given either alone or in combination with radiotherapy, surgery, or both as neoadjuvant, concomitant, or adjuvant treatment. Improved treatments have resulted in prolonged survival and better control of disease and treatment related complications. The majority of patients will, however, still face a range of symptoms and side effects such as nausea, vomiting, pain, insomnia, appetite loss, and fatigue. Surveys have shown that fatigue is among the most frequent and burdensome side effect of chemotherapy and results in impaired or diminished physical activity. ${ }^{1}$ Whereas most side effects are drug specific, fatigue is associated not only with most antineoplastic drugs but also with the disease itself. ${ }^{2}$ Prevention and treatment of fatigue are complicated; treatment with drugs alone is rarely adequate. In addition, psychosocial problems often follow the diagnosis of cancer and subsequent chemotherapy. ${ }^{3}$ For some patients, diagnosis and treatment are synonymous with an inactive daily life, ${ }^{4}$ resulting in loss of muscle mass and strength. ${ }^{56}$ Exercise training has been introduced to improve physical capacity and quality of life 
and to reduce fatigue. Few intervention studies have included patients who were undergoing chemotherapy and the evidence is modest. ${ }^{7-9}$

Exercise studies have included predominantly women with breast cancer after cytostatic treatment and the interventions provided improved physical fitness and psychological benefits. ${ }^{7-9}$ Typically studies investigated the effects of a single activity of moderate intensity, such as cardiovascular training on stationary bicycles, rather than resistance training. Additional studies are needed to provide evidence on whether patients with different cancer diagnoses, stages of disease, and symptoms can benefit from combined resistance and cardiovascular training when undergoing chemotherapy. Moreover, high intensity exercise might further improve the patients' physical capacity, as recorded in sports science studies including healthy adults. $^{510}$

A wide range of low intensity interventions comprising psychosocial activities (relaxation and massage) are recommended as adjuvants to pharmacological therapies, to relieve nausea, pain, and fatigue and to increase the patient's perception of self control. ${ }^{1112}$

This body of research provides evidence that exercise and psychosocial interventions might be beneficial when tested as separate components. We developed a multimodal intervention consisting of high and low intensity components to achieve a broad spectrum of effects. The intervention was designed to appeal to male and female patients with cancer, irrespective of diagnosis and stage of disease.

The objective of the present study was to investigate the effect of a six week supervised structured group intervention comprising high intensity physical training and low intensity training in an intervention group compared with a control group. We tested the hypothesis that the intervention, as an adjunct to conventional care, could reduce fatigue (primary outcome), and improve other side effects, general physical and emotional wellbeing, global health status or quality of life, physical capacity, and physical activity in men and women with cancer who were undergoing adjuvant chemotherapy or treatment for advanced disease.

\section{METHODS}

Recruitment and assignment

Participants were recruited from the departments of oncology and haematology at Rigshospitalet and Herlev Hospital (Copenhagen University Hospitals) from March 2004 to March 2007. Participants were eligible if they had a diagnosis of cancer, had received at least one cycle of chemotherapy for advanced disease or as adjuvant treatment, had a WHO performance status of 0 or 1, and were aged 18-65 years. Exclusion criteria were brain or bone metastases, thrombocytopenia $\left(<50 \times 10^{9} / 1\right)$, myocardial infarction within the past three months, or uncontrolled hypertension (diastolic pressure $>95 \mathrm{~mm} \mathrm{Hg}$ ). The patients were approached and enrolled by research nurses or by physicians and nurses from eight treatment departments. Furthermore, the participants could contact the project team directly; information was accessible on posters and pamphlets in the hospitals' inpatient and outpatient departments.

After written informed consent, the baseline measures were obtained from the participants, who were randomly allocated to an intervention or a control group. The patients were stratified by sex, diagnosis category (breast, bowel, other oncological malignancies, haematological malignancies), and disease status (no evidence of disease or evidence of disease). Patients with no evidence of disease received adjuvant treatment after radical local treatment for their cancer disease, while patients with evidence of disease had residual or advanced disease after the initial diagnosis of cancer was made by biopsy or local treatment. Randomisation was done by computer (Clinical Internet Trial Management System: CITMAS). The allocation sequence was executed by the clinical research unit and concealed from the project team. The data were collected by a physiotherapist and a trained nurse specialist, who also conducted the daily training sessions. Data were anonymised by use of an identification code; administrative data were kept in a separate database. Blinding the participants to group assignment was not possible. Outcome measures were keyed in and analysed by research assistants who were not involved with the participants.

Intervention: exercise programme

Participants assigned to the intervention group received standard medical care while participating in a group based multimodal high and low intensity exercise intervention (supervised by trained nurse specialists and physiotherapists). There were seven to 10 participants per group, each group including individuals of both sexes and with various diagnoses and disease statuses.

On Mondays, Wednesdays, and Fridays, the patients participated in high intensity physical training for 90 minutes followed by 30 minutes relaxation training. On Tuesdays the programme included 90 minutes of body awareness training followed by 30 minutes of relaxation training. Mondays and Fridays were reserved for the patients to receive 30 minutes of massage. The intervention took place in a fitness facility at Rigshospitalet, and was undertaken over a six week period for nine hours per week. The multimodal intervention constituted a total package; the participants could not select one activity in preference over another. The intervention consisted of high and low intensity activities equivalent to a total of 43 metabolic equivalent of task (MET) hours per week. ${ }^{13}$ The high intensity physical training sessions comprised three components: 30 minutes of warm-up exercises, 45 minutes of resistance training, and 15 minutes of cardiovascular training. The warm-up consisted of dynamic exercises with the large muscle groups, along with balance and coordination training, and had an estimated average intensity of 9 METs (4.5 MET hours per training session). Six machines were used for resistance training: a leg press, a chest 
press, a pull down, an abdominal crunch, a lower back, and a knee extension (Technogym, Gambettola, Italy). The one repetition maximum test was used to measure the weight a patient could lift once on any specific machine. The weight was expressed as $100 \%$ of the patient's strength and a training programme was then developed based on that measure. The aim of the resistance training component was to accomplish three continuous series of five to eight repetitions at 70 $100 \%$ of the one repetition maximum test. ${ }^{14}$ To ensure progression, all patients performed one repetition maximum test every other week, with subsequent adjustments made to their training programmes. Resistance training was estimated to have an intensity of 5.5 METs (4 MET hours per training session). Cardiovascular training involved interval training on stationary bicycles with a workload of 70-250 W, equivalent to $85-95 \%$ of each participant's maximum heart rate. This training was estimated to have an intensity of 15 METs (3.75 MET hours per training session).

Low intensity physical training comprised three psychosocial components: relaxation (30 minutes four times a week), body awareness and restorative training (90 minutes once a week), and massage (30 min twice a week). In relaxation sessions, the participants lay on mats with pillows and blankets and were instructed in the use of relaxation mechanisms, using principles of progressive muscle relaxation. Participants were instructed in tensing and relaxing major muscle groups, working from head to foot. ${ }^{15}$ They were asked to focus on the contrast in sensations between muscle tension and relaxation. Audiotape progressive muscle relaxation was used to enhance treatment integrity by assuring that all participants were exposed to the same instructions. Body awareness and restorative training adopted various themes that focused on the participants' awareness, recognition, and control of their bodies. The first session was dedicated to stretching, which can contribute to reducing discomfort and pain. ${ }^{16}$ The theme selected for weeks 2 and 3 was respiration, inspired by yoga, which aims to increase mental and physical energy. ${ }^{17}$ The remaining weeks (weeks 4-6) focused on postural awareness and movement re-education, based on Pilates techniques. ${ }^{18}$ Massage was relaxing, facilitative, or therapeutic, including scar tissue massage. A few patients requested venous pump massage or ultrasound therapy. ${ }^{19}$ Relaxation training and massage were each estimated to have an intensity of $1 \mathrm{MET}$ (total of 3 MET hours per week), while body awareness and restorative training were estimated to have an intensity of 2.5 METs (3.25 MET hours per week). ${ }^{13}$

\section{Safety}

Pre-exercise screening was performed before high intensity physical training. If one of the following criteria was met, the participant was excluded from the physical training component of the programme on that specific day: diastolic blood pressure $<45 \mathrm{~mm}$ $\mathrm{Hg}$ or $>95 \mathrm{~mm} \mathrm{Hg}$; pulse at rest $>100$ beats per minute; temperature $>38^{\circ} \mathrm{C}$; respiration frequency $>20$ per minute; infections requiring treatment with antibiotics; ongoing bleeding; fresh petechiae; bruises; B thrombocytes $<50 \times 10^{9} / 1$; B leucocytes $<1.0 \times 10^{9} / 1$. During the training sessions the participants' heart rate was continuously monitored by means of a wireless heart rate transmitter, and the participants were advised to respect their own physical limitations.

\section{Control group}

Participants assigned to the control group received conventional medical care, and completed outcome measures in parallel with the intervention group. The control group was allowed to freely increase physical activity. They were invited to participate in the exercise programme after the six week assessments.

\section{Primary and secondary outcome measures}

Assessment included patient rated and objectively measured physiological outcomes. All outcomes were assessed at baseline and after intervention. The primary outcome, fatigue, was assessed using the European Organization for Research and Treatment of Cancer Quality of Life Questionnaire (EORTC QLQ-C30; 30 items). ${ }^{20}$ The EORTC QLQ-C30 comprises five functional scales, nine symptom scales or items, and a global health status/quality of life scale. All scale/single item measures range in score from 0100. A high score on the functional and global health status/quality of life scale represents a high or healthy level of functioning and high quality of life, while a high score for a symptom scale represents a high level of symptomatology. Secondary outcomes included all other scales on EORTC QLQ-C30, and general wellbeing was further assessed with the Medical Outcomes Study Short Form (MOS SF-36), ${ }^{21}$ which contains eight scales measuring general health concepts and two summary scales: physical component scale, and mental component scale.

Leisure time physical activity level was explored by questionnaire. The participants were classified as: sedentary (completely inactive); walking or cycling for pleasure; regular physical exercise at least 3 hours a week; or intense physical activity more than 4 hours a week. $^{22-24}$

Other secondary outcomes included muscular strength stated in kilograms and aerobic capacity $\left(\mathrm{Vo}_{2} \mathrm{max}\right)$ stated in $\mathrm{l} / \mathrm{min}$. Muscular strength was ascertained using the one repetition maximum test to evaluate upper and lower body strength. ${ }^{25}$ The tests were performed on Technogym (Gambettola, Italy) variable resistance equipment (chest press, leg press, and pull down) and targeted large muscular groups. Each patient started the test with three to four repetitions on each machine, on a low weight. The patient would increase the weight by $2.5 \mathrm{~kg}$ continuously. The test was complete when the patient reached maximum capacity (one repetition maximum). To obtain an overall score for improvement in muscular strength we used the mean of the relative changes in strength in chest press, leg press, and pull down. 
$\mathrm{Vo}_{2}$ max was indirectly estimated by use of a stepwise work capacity on a stationary exercise cycle (Monark Ergomedic 839E, Sweden). The steady rate test started with a workload of $67 \mathrm{~W}$ over 8 minutes with the patients' pulse rate being recorded during the final minute of the test. The watt max test started at $67 \mathrm{~W}$, increasing by $20 \mathrm{~W}$ with each consecutive minute until the patient did not wish to continue cycling. $\mathrm{Vo}_{2} \max$ was estimated using the formula $\mathrm{Vo}_{2} \max =0.16+(0.0117 \times \mathrm{MPO})$ where maximal power out (MPO) was measured in watts. ${ }^{26}$

To compare estimated $\mathrm{Vo}_{2}$ max with the maximum oxygen consumption both measurements were done in seven patients, and no significant difference was found between the tests. ${ }^{27} \mathrm{We}$ chose to use estimated $\mathrm{Vo}_{2}$ max because this method was feasible for use in the study population.

Demographic and behavioural data were collected by self report, and medical data were drawn from records.

\section{Sample size calculation and statistical analysis}

According to the scoring manual for EORTC QLQC30 a change of 10 points or more is considered to be a moderate to large clinically significant change. ${ }^{20}$ Based on this assumption and our pilot study $(n=82)^{28}$ the sample size was calculated on an $80 \%$ power to detect a difference in score of 10 points (SD 25) on the EORTC QLQ-30 fatigue scale (items 10,12, and 18 ), using a type 1 error of $5 \%$, a type 2 error of $20 \%$, and univariate analysis ( $t$ test). This calculation resulted in 98 participants in each group. To accommodate expected dropout before study completion, because of inclusion of participants with present evidence of disease, a total of 135 participants were included in each group.

Baseline comparisons were done using univariate analyses of variance for continuous variables. For categorical measures likelihood ratio based $\chi^{2}$ test for symmetry and marginal homogeneity was used to evaluate the potential changes.

The main analysis examined whether differences in outcome (mean differences) between baseline and six weeks existed between the control and intervention groups in all outcome measures (EORTC QLQ-C30, MOS SF-36, $\mathrm{Vo}_{2} \mathrm{max}$, and muscular strength). We performed a forward stepwise regression analysis using differences in outcome between baseline and six weeks (in all outcome measures) as the dependent variable in a general linear model. The stepwise procedure began by identifying the covariate that was most strongly related to the dependent variable. The next strongest related covariate was then selected after controlling for the first covariate, and so on. The variable intervention/control was fixed and the following covariates were tested: sex, age, cohabitation, educational level, baseline outcome score, relative change in $\beta$ haemoglobin, $\mathrm{Vo}_{2} \max$, one repetition maximum knee extension, and the five disease related covariatesdiagnosis, evidence of disease, relapse of disease, and chemotherapy cycles before and during the study period. Potential effect modifications (interactions) of the five disease related covariates and their effect on the estimated mean difference were also tested.

All analyses were tested with a significance level of $\mathrm{P}<0.05$ by using the intention to treat principle. Available data for participants with missing data were included under the "missing at random" assumption.

Clinically important changes were estimated as effect sizes using Cohen's guidelines, whereby a value of 0.2 denotes a small, 0.5 a medium, and 0.8 a large effect size. ${ }^{29-31}$ Effect size was calculated by the mean difference divided by the pooled standard deviation, the root mean square error estimated from the general linear model.

Participants classified as lost to follow-up ( $n=34)$ were compared with the study group $(n=235)$ for baseline demographic data using ordinary $t$ test and ${ }^{2} \chi^{2}$ test for homogeneity.

Data were entered into Excel using Microsoft Office 2000 Professional for Windows XP and SAS (version 9.1.3) software for statistical analysis.

\section{RESULTS}

\section{Participants}

During the study period (March 2004 to March 2007), 1956 patients with cancer aged 18-65 years were referred for chemotherapy (fig 1). Two hundred and sixty nine patients met the inclusion criteria and agreed to participate. For the primary outcome (fatigue) we obtained post-intervention data from 118 participants in the intervention group (87.4\%) and 117 in the control group (87.3\%). The intervention group adherence rate was $70.8 \%$ (17 of 24 training days, range 3-24) of their supervised exercise sessions.

The control and intervention groups were matched at baseline for demographic and medical characteristics (table 1). Participants were on average 47 years old (range 20-65 years), and 73\% were female. The study included patients with 21 different cancer diagnoses: 17 with solid tumours and four with malignant haematological diseases. Forty eight per cent had evidence of disease and 52\% no evidence of disease. The median number of days since diagnosis was 84 .

The participants received a total of 59 different chemotherapy regimens during the study period. The most frequent chemotherapy regimen included cyclophosphamide, epirubicin, and 5-fluorouracil and was administered to 49 patients in each group. Also frequent were 5-fluorouracil based regimens in patients with colorectal cancer (12 in the control group and 14 in the intervention group) and platinum based regimens in women with ovarian cancer $(10 v 15)$ and in men with testicular cancer (eight $v$ six). All other regimens were given to fewer than five patients and regimens were balanced between groups. The intervention and control groups had received a mean of 2.5 and 2.6 cycles of chemotherapy, respectively, before the study period, and received a mean of 1.9 and 1.8 cycles, respectively, during the six week study period (table 2). 


\section{Main outcomes}

Changes in patient rated outcomes

Tables 3 and 4 present the patient rated outcomes measured at baseline and after six weeks for the intervention and the control groups.

We found a significant effect in favour of the intervention group for the primary outcome, fatigue, in EORTC QLQ-C30. The fatigue score was reduced in the intervention group by an estimated mean difference of -6.6 points $(95 \% \mathrm{CI}-12.3$ to -0.9$)$ compared with the control group $(\mathrm{P}=0.02$, effect size $=0.33$, 95\% CI 0.04 to 0.61 ) (table 3).

We noted significant effects of the intervention on seven of 10 subscales on general wellbeing (MOS SF36): physical functioning (effect size $0.37,95 \%$ CI 0.09 to 0.65$)$, role physical $(0.37,0.10$ to 0.64$)$, vitality $(0.55$, 0.27 to 0.82$)$ role emotional $(0.32,0.05$ to 0.59$)$, mental health $(0.28,0.02$ to 0.56$)$, physical component scale $(0.35,0.06$ to 0.63$)$, and mental component scale $(0.41,0.14$ to 0.69$)$ (table 4$)$. Significant interaction was seen for role physical, with greater improvement in patients with no evidence of disease than in those with evidence of disease.

\section{Changes in objectively measured physiological outcomes}

$\mathrm{Vo}_{2}$ max increased more in the intervention group than in the control group (table 5); the mean improvement in $\mathrm{Vo}_{2} \max$ was $10.7 \%$ (SD 0.5) compared with no change in the control group. Significant improvements in muscular strength were seen in favour of the intervention group. The average improvement in muscular

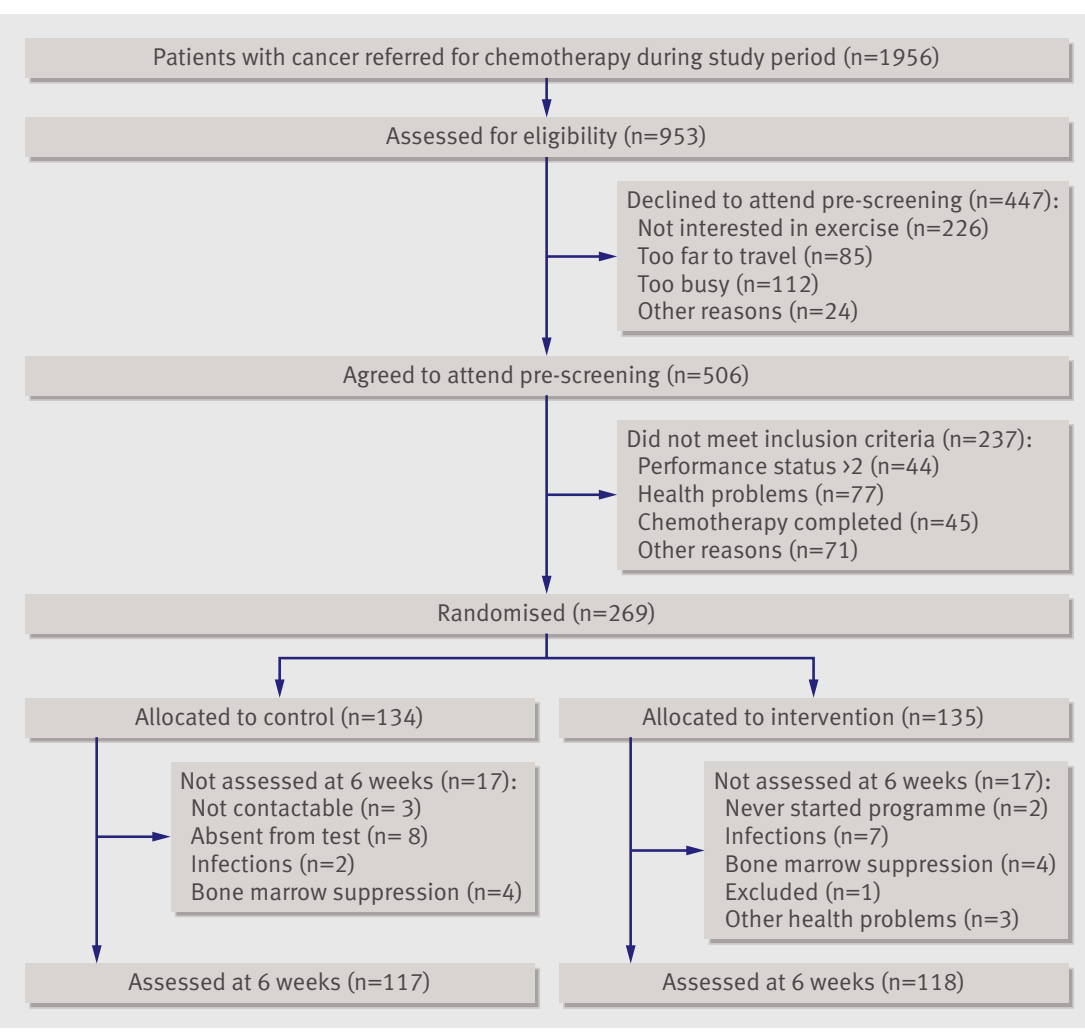

Fig 1| Trial profile strength was $29.6 \%$ (SD 36.4) for the intervention group (table 5). Significant interaction was seen for the muscular strength chest press, with more improvement in patients with no evidence of disease than in those with evidence of disease.

\section{Changes in self reported physical activity level}

Sixty-six per cent of the study population reported physical activity of at least 3 hours per week before their illness, whereas at baseline $18 \%$ had a sedentary lifestyle, when undergoing chemotherapy (table 1); only $33 \%$ of the population reported physical activity of at least 3 hours per week. Homogeneity of the marginal distribution was not fulfilled $(\mathrm{P}<0.0001)$; the level of self reported physical activity fell during the period from pre-illness to baseline for both the intervention and control group.

In the control group the joint distribution of baseline and post intervention physical activity satisfied symmetry $(\mathrm{P}=0.4)$ and as such homogeneity of the marginal distribution, indicating that the level of physical activity level had not changed during the period. By contrast, neither the hypothesis of symmetry nor marginal homogeneity was fulfilled $(\mathrm{P}<0.0001$ for both hypotheses) for the intervention group. Post intervention, the patients increased their activity level. The mode changed from "walking or cycling for pleasure" $(40 \%)$ at baseline to"regular physical exercise" (53\%) after intervention.

\section{DISCUSSION}

\section{Principal findings}

Men and women with a broad range of ages, cancer diagnoses, disease statuses, and chemotherapy regimens participated in this study. The multimodal intervention of high intensity exercise, relaxation, body awareness training, and massage for patients undergoing chemotherapy showed broad effects. We noted significant effects on fatigue, vitality, physical functioning, role functioning, role emotional, mental health, physical component scale, and mental component scale, physical capacity $\left(\mathrm{VO}_{2} \max\right.$ and muscular strength), and physical activity levels, while global health status/quality of life and symptom scales did not show improvements.

The intervention was offered as a package, and it must be viewed as an entity, whereby each component has had a role in the outcomes. Our findings confirmed that both men and women benefited from training in mixed groups, and no differences between men and women were observed in the dropout rate.

The primary outcome, fatigue, was the most frequently reported symptom; $65 \%$ of the study population reported a fatigue level greater than that of the general population (mean $>20$ ) at baseline ${ }^{31}$ and $29 \%$ reported severe fatigue (mean $>60$ ).

The effect size of the improvement in fatigue (0.33) suggests a small to medium clinically important change. $^{293032}$ Our result differs from findings of a meta-analysis which indicated that the magnitude of 
Table 1|Demographic and medical characteristics, and physical activity level for all patients and by group assignment. Values are numbers (percentages) of patients unless stated otherwise

Control group $(\mathrm{n}=134) \quad$ Intervention group $(\mathrm{n}=135)$

Demographic characteristics

Age, years

\begin{tabular}{|c|c|c|}
\hline Mean (SD) & $47.2(10.6)$ & $47.2(10.7)$ \\
\hline Range & $20-65$ & $21-65$ \\
\hline Married, cohabiting, or in a relationship & $89(66.9)$ & $95(70.4)$ \\
\hline \multicolumn{3}{|l|}{ Sex } \\
\hline Male & $39(29.1)$ & $34(25.2)$ \\
\hline Female & $95(70.9)$ & $101(74.8)$ \\
\hline Completed secondary school or higher & $106(79.7)$ & $104(77.0)$ \\
\hline Current smoker & $19(14.3)$ & $26(19.3)$ \\
\hline \multicolumn{3}{|l|}{ Medical characteristics } \\
\hline Median (range) days since diagnosis & $89.5(31-271)$ & $83(34-280)$ \\
\hline No evidence of disease (NED) baseline & $72(53.7)$ & $68(50.4)$ \\
\hline Evidence of disease (ED) baseline & $62(46.3)$ & 67 (49.6) \\
\hline Relapsed disease & $26(19.4)$ & $17(12.6)$ \\
\hline Mean (SD) $\beta$ haemoglobin, mmol/l & $7.90(0.8)$ & $7.95(0.8)$ \\
\hline Cancer of breast (NED/ED) & $59(50 / 9)$ & $60(49 / 11)$ \\
\hline Cancer of bowel (NED/ED) & $17(10 / 7)$ & $18(13 / 5)$ \\
\hline \multicolumn{3}{|l|}{ Oncological malignancies (NED/ED) } \\
\hline Cancer of ovaries & $11(3 / 8)$ & $16(5 / 11)$ \\
\hline Cancer of testes & $9(0 / 9)$ & $7(0 / 7)$ \\
\hline Cancer of oesophagus & $3(0 / 3)$ & $2(0 / 2)$ \\
\hline Cancer of brain & $4(0 / 4)$ & $1(0 / 1)$ \\
\hline Cancer of cervix & $2(0 / 2)$ & $4(0 / 4)$ \\
\hline Cancer of pharynx & $1(0 / 1)$ & $2(0 / 2)$ \\
\hline Cancer of pancreas & $2(0 / 2)$ & $1(0 / 1)$ \\
\hline Cancer of stomach & $2(0 / 2)$ & $1(0 / 1)$ \\
\hline Other diagnoses & $9(3 / 6)$ & $10(1 / 9)$ \\
\hline Haematological malignancies (NED/ED) & $15(6 / 9)$ & $13(0 / 13)$ \\
\hline Hodgkin's & 4 & 6 \\
\hline Non-Hodgkin's lymphoma & 5 & 7 \\
\hline Acute leukaemia & 5 & 0 \\
\hline Chronic leukaemia & 1 & 0 \\
\hline \multicolumn{3}{|l|}{ physical activity level } \\
\hline \multicolumn{3}{|l|}{ Pre-illness } \\
\hline Sedentary & $5(4.0)$ & $10(7.5)$ \\
\hline Walking or cycling for pleasure & $34(27.0)$ & $40(30.1)$ \\
\hline Regular physical exercise, at least $3 \mathrm{~h} /$ week & $75(59.5)$ & $74(55.6)$ \\
\hline Intense physical activity, more than $4 \mathrm{~h} /$ week & $12(9.5)$ & $9(6.8)$ \\
\hline \multicolumn{3}{|l|}{ Baseline } \\
\hline Sedentary & $25(19.8)$ & $23(17.4)$ \\
\hline Walking or cycling for pleasure & $48(38.1)$ & $75(56.8)$ \\
\hline Regular physical exercise, at least $3 \mathrm{~h} /$ week & $47(37.3)$ & $30(22.7)$ \\
\hline Intense physical activity, more than $4 \mathrm{~h} /$ week & $6(4.8)$ & $4(3.0)$ \\
\hline
\end{tabular}

effect from exercise on cancer related fatigue might be too small to be clinically meaningful (effect size $=0.13$, 95\% CI -0.06 to 0.33$).{ }^{7}$ A Cochrane meta-analysis found the association between exercise and fatigue to be insignificant and inconclusive owing to lack of studies. ${ }^{9}$ Likewise, two recent exercise studies of moderate intensity in women with breast cancer receiving adjuvant chemotherapy found no significant improvements in fatigue. ${ }^{334}$
Table 2 |Chemotherapy cycles and surgery, by group assignment. Values are numbers of patients unless stated otherwise

\begin{tabular}{lccc} 
Chemotherapy cycles & $\begin{array}{c}\text { Control } \\
\text { group } \\
(\mathbf{n}=134)\end{array}$ & $\begin{array}{c}\text { Intervention } \\
\text { group } \\
(\mathbf{n}=135)\end{array}$ & P value \\
\hline Before study period & - & - & 0.75 \\
\hline 1 cycle & 33 & 41 & \\
\hline 2 cycles & 43 & 37 & \\
\hline 3 cycles & 25 & 21 & \\
\hline 4 cycles & 16 & 19 & \\
\hline >4 cycles & 17 & 17 & \\
\hline Mean & 2.6 & 2.5 & \\
\hline During study period & - & - & \\
\hline 1 cycle & 29 & 39 & \\
\hline 2 cycles & 96 & 89 & \\
\hline 2 cycles & 9 & 7 \\
\hline Mean & 1.8 & 1.9 \\
\hline Advanced/adjuvant & $72 / 62$ & $73 / 62$ \\
\hline Surgery & 109 & 109 \\
\hline Mastectomy & 39 & 33 \\
\hline Lumpectomy & 19 & 25 \\
\hline Hysterectomy & 11 & 16 \\
\hline Ileo-colostomy & 5 & 3 \\
\hline Orchiectomy & 8 & 6 \\
\hline Other & 27 & 26 \\
\hline
\end{tabular}

The cause of fatigue in cancer patients remains nebulous and multifactorial. Patients report fatigue as a state of physical disturbance and loss of function, with exhaustion being the lead factor in reduced physical activity. Severe fatigue results from extreme muscular de-conditioning caused by both the disease and treatment but can also be triggered by a sedentary lifestyle. ${ }^{35}$ However, only $18 \%$ of the study population had a sedentary lifestyle at baseline, which may indicate that theirfatigueburdenwas primarily duetothedisease or to the chemotherapy. The patients' level of fatigue (mean 39.7, SD 25.8) corresponded to those obtained in other studies investigating cancer patients with the same WHO performance status and severity of disease (mean 40, SD 26). ${ }^{32}$

Although the intervention reduced the patients' levels of fatigue after six weeks (mean 34.6, SD 24.3) the scores after intervention did not reach the level of the general population (mean 21).

Our qualitative research with participants from the pilot study showed that a reduction in fatigue could not be interpreted as an overall improvement in quality of life. However, a complete, partial, or periodic reduction in fatigue affected the patients' daily lives. ${ }^{36}$

Consistent with recently published studies and metaanalyses on exercise interventions, including those in women receiving adjuvant therapy for breast cancer $^{7-93334}$ we found no significant improvements in global health status/quality of life (EORTC QLQC30), which incorporates two questions: "How would 
you rate your overall health during the past week?" and "How would you rate your overall quality of life during the past week?"

Table $3 \mid$ Health related Quality of Life (EORTC QLQ-C30) outcome variables and estimated differences

\begin{tabular}{|c|c|c|c|c|}
\hline \multirow[b]{2}{*}{ Outcome variable } & \multicolumn{2}{|c|}{ Mean (SD) } & \multicolumn{2}{|c|}{ Test (reference: control) } \\
\hline & Baseline & 6 weeks & $\begin{array}{l}\text { Estimated mean } \\
\text { difference }(95 \% \mathrm{Cl})\end{array}$ & $P^{*}$ \\
\hline \multicolumn{5}{|l|}{ Maximum number } \\
\hline Control & 134 & 117 & NA & NA \\
\hline Intervention & 135 & 118 & NA & NA \\
\hline \multicolumn{5}{|c|}{ Global health status/quality of life } \\
\hline Control & $60.2(22.4)$ & $63.3(22.4)$ & & \\
\hline Intervention & $63.8(21.1)$ & $67.2(20.3)$ & $2.2(-2.7$ to 7.1$)$ & 0.4 \\
\hline \multicolumn{5}{|l|}{ Physical functioning } \\
\hline Control & $84.0(15.7)$ & $86.4(14.5)$ & & \\
\hline Intervention & $84.7(14.5)$ & $89.0(12.4)$ & $2.4(-0.4$ to 5.1$)$ & 0.09 \\
\hline \multicolumn{5}{|l|}{ Role functioning } \\
\hline Control & $65.6(28.5)$ & $68.9(26.5)$ & & \\
\hline Intervention & $68.7(28.4)$ & $74.8(26.3)$ & $4.6(-1.7$ to 10.9$)$ & 0.2 \\
\hline \multicolumn{5}{|c|}{ Emotional functioning } \\
\hline Control & $75.7(19.3)$ & $80.6(17.8)$ & & \\
\hline Intervention & $77.6(12.2)$ & $81.3(17.2)$ & $-0.3(-4.0$ to 3.4$)$ & 0.9 \\
\hline \multicolumn{5}{|c|}{ Cognitive functioning } \\
\hline Control & $81.9(17.8)$ & $81.3(19.8)$ & & \\
\hline Intervention & $84.4(17.1)$ & $83.8(16.7)$ & $1.7(-2.6$ to 6.0$)$ & 0.4 \\
\hline \multicolumn{5}{|l|}{ Social functioning } \\
\hline Control & $78.6(21.2)$ & $79.4(20.8)$ & & \\
\hline Intervention & $83.3(20.6)$ & $82.6(20.5)$ & $4.5(-1.4$ to 10.3$)$ & 0.1 \\
\hline \multicolumn{5}{|l|}{ Fatigue } \\
\hline Control & $43.0(23.9)$ & $41.0(22.7)$ & & \\
\hline Intervention & $39.7(25.8)$ & $34.6(24.3)$ & $-6.6(-12.3$ to -0.9$)$ & 0.02 \\
\hline \multicolumn{5}{|c|}{ Nausea and vomiting } \\
\hline Control & $17.3(21.7)$ & $13.7(18.4)$ & & \\
\hline Intervention & $16.0(22.9)$ & $13.4(17.3)$ & $-0.6(-5.2$ to 3.9$)$ & 0.8 \\
\hline \multicolumn{5}{|l|}{ Pain } \\
\hline Control & $15.4(22.7)$ & $16.8(20.6)$ & & \\
\hline Intervention & $17.4(22.4)$ & $14.6(17.1)$ & $-2.9(-7.6$ to 1.7$)$ & 0.2 \\
\hline \multicolumn{5}{|l|}{ Dyspnoea } \\
\hline Control & $18.2(25.3)$ & $18.2(24.4)$ & & \\
\hline Intervention & $15.3(21.6)$ & $14.3(21.5)$ & $-2.9(-8.6$ to 2.7$)$ & 0.3 \\
\hline \multicolumn{5}{|l|}{ Insomnia } \\
\hline Control & 32.7 (31.5) & $26.4(27.5)$ & & \\
\hline Intervention & $22.7(26.9)$ & $18.4(23.9)$ & $-3.5(-9.5$ to 2.6$)$ & 0.3 \\
\hline \multicolumn{5}{|l|}{ Appetite loss } \\
\hline Control & $16.5(26.6)$ & $14.6(22.1)$ & & \\
\hline Intervention & $18.4(27.6)$ & $12.8(22.3)$ & $-3.4(-8.9$ to 2.0$)$ & 0.2 \\
\hline \multicolumn{5}{|l|}{ Constipation } \\
\hline Control & $22.2(29.5)$ & $18.4(25.3)$ & & \\
\hline Intervention & $16.7(24.0)$ & $20.8(39.4)$ & $4.5(-4.0$ to 13.1$)$ & 0.3 \\
\hline \multicolumn{5}{|l|}{ Diarrhoea } \\
\hline Control & $14.0(23.5)$ & $13.0(21.9)$ & & \\
\hline Intervention & $14.8(28.0)$ & $17.0(26.1)$ & $4.1(-1.7$ to 10.0$)$ & 0.2 \\
\hline \multicolumn{5}{|l|}{ Financial difficulties } \\
\hline Control & $14.3(27.7)$ & $13.0(25.9)$ & & \\
\hline Intervention & $11.1(21.5)$ & $10.8(19.3)$ & $-0.3(-5.0$ to 4.4$)$ & 0.9 \\
\hline
\end{tabular}

*Adjusted general linear model.
Courneya et al tested resistance versus aerobic exercise for the duration of the patients' chemotherapy (17 weeks) in 242 patients with breast cancer. Neither aerobic exercise nor resistance training significantly improved cancer specific quality of life (as measured by functional assessment of cancer therapy questionnaire - anaemia). ${ }^{34}$ Mutrie et al conducted a 12 week supervised group exercise programme during chemotherapy for early stage breast cancer in 203 women, and no significant effect was seen for general quality of life (functional assessment of cancer therapy questionnaire - general). ${ }^{33}$

The failure of the intervention to significantly improve global health status/quality of life indicates that this type of short term intervention was not able to overcome the complexity of patients' overall negatively affected situation. Being diagnosed with cancer and exposed to chemotherapy disrupts the patient's life, affecting physiological and psychological functioning and contributing to negative effects on the global health status/quality of life. ${ }^{37} 38$ Improvements in this measure may have been too ambitious a goal in this short term clinical trial.

The intervention showed no significant effect on seven of eight somatic symptom scales in the EORTC QLQ-C30 questionnaire. This finding might because many side effects induced by chemotherapy can be prevented or treated by supportive care drugs; for example, evidence based guidelines exist for the prevention and treatment of nausea, vomiting, and diarrhoea. $^{39}$

Supportive medical treatment may consequently have caused the observed floor effect where 50-70\% of the study population were placed in the lowest quartiles on six of the symptom scales. Similarly we found a ceiling effect on three EORTC QLQ-C-30 functional scales.

By contrast, no such floor-ceiling effect was seen when applying the general wellbeing MOS SF-36 scale - which might be a more sensitive tool than the others for measuring effects of exercise in cancer patients - a finding confirmed in other exercise intervention studies. ${ }^{110}$

Significant effects of the intervention were recorded for seven of 10 subscales for general wellbeing (MOS SF-36), with small to medium effect sizes for six of the scales. The present multimodal intervention showed greater significant effects and higher mean differences on several scales (MOS SF-36) compared to a 26 week supervised aerobic exercise programme in breast cancer patients receiving less toxic chemotherapy. ${ }^{40}$ Particularly, vitality showed an effect size that was greater than medium (0.55). In existing meta-analyses regarding exercise based interventions, fatigue and quality of life are often highlighted, while measurement of vitality and its association with fatigue are not..$^{7-9}$ In the present study an explorative general linear model analysis demonstrated that changes in fatigue were strongly affected by an increase in vitality. This finding may suggest that the multimodal intervention including a high intensity component generates vitality and 
Table 4 | General wellbeing (MOS SF-36) outcome variables and estimated differences

\begin{tabular}{|c|c|c|c|c|}
\hline \multirow[b]{2}{*}{ Outcome variable } & \multicolumn{2}{|c|}{ Mean (SD) } & \multicolumn{2}{|c|}{ Test (reference: control) } \\
\hline & Baseline & 6 weeks & $\begin{array}{c}\text { Estimated mean } \\
\text { difference }(95 \% \mathrm{Cl})\end{array}$ & $\mathrm{P}^{*}$ \\
\hline \multicolumn{5}{|l|}{ Maximum number } \\
\hline Control & 134 & 117 & NA & NA \\
\hline Intervention & 135 & 118 & NA & NA \\
\hline \multicolumn{5}{|l|}{ Physical functioning } \\
\hline Control & $83.6(14.8)$ & $84.3(16.2)$ & & \\
\hline Intervention & $84.3(13.7)$ & $88.2(13.2)$ & $4.4(1.1$ to 7.7$)$ & 0.01 \\
\hline \multicolumn{5}{|l|}{ Role physical } \\
\hline Control & $27.1(35.7)$ & $31.8(37.6)$ & & \\
\hline Intervention & $30.5(35.2)$ & $46.1(40.2)$ & 12.4 (3.4 to 21.5$)$ & 0.007 \\
\hline \multicolumn{5}{|l|}{ Bodily pain } \\
\hline Control & $74.0(24.6)$ & $75.7(22.7)$ & & \\
\hline Intervention & $70.9(27.6)$ & $77.6(20.0)$ & $3.4(-1.2$ to 8.0$)$ & 0.2 \\
\hline \multicolumn{5}{|c|}{ General health perceptions } \\
\hline Control & $61.1(22.9)$ & $65.5(22.4)$ & & \\
\hline Intervention & $64.9(18.4)$ & $68.7(19.7)$ & $0.6(-3.7$ to 4.9$)$ & 0.8 \\
\hline \multicolumn{5}{|l|}{ Vitality } \\
\hline Control & $55.8(21.1)$ & $55.6(21.6)$ & & \\
\hline Intervention & $57.8(20.2)$ & $65.5(18.1)$ & $8.8(4.4$ to 13.1$)$ & $<0.0001$ \\
\hline \multicolumn{5}{|l|}{ Social functioning } \\
\hline Control & $75.4(21.8)$ & $76.5(22.0)$ & & \\
\hline Intervention & $77.0(21.1)$ & $79.7(22.2)$ & $2.4(-2.8$ to 7.6$)$ & 0.4 \\
\hline \multicolumn{5}{|l|}{ Role emotional } \\
\hline Control & $58.6(41.2)$ & $58.7(41.9)$ & & \\
\hline Intervention & $56.1(39.0)$ & $69.6(40.1)$ & $12.0(1.9$ to 22.0$)$ & 0.02 \\
\hline \multicolumn{5}{|l|}{ Mental health } \\
\hline Control & $72.0(16.7)$ & $74.2(16.1)$ & & \\
\hline Intervention & $74.0(16.3)$ & $78.6(15.0)$ & $3.3(0.2$ to 6.4$)$ & 0.04 \\
\hline \multicolumn{5}{|c|}{ Physical component scale } \\
\hline Control & $44.3(8.3)$ & $45.1(8.5)$ & & \\
\hline Intervention & $44.2(8.4)$ & $47.4(6.7)$ & $1.9(0.3$ to 3.4$)$ & 0.02 \\
\hline \multicolumn{5}{|c|}{ Mental component scale } \\
\hline Control & $46.9(10.2)$ & $47.3(10.0)$ & & \\
\hline Intervention & $46.5(9.7)$ & $50.5(9.4)$ & $3.2(1.1$ to 5.4$)$ & 0.004 \\
\hline
\end{tabular}

thereby reduces fatigue. The patients' perception of improved vitality is important especially during chemotherapy treatment periods. ${ }^{36}$

At baseline, the patients had a mean score of 30.5 (SD 35.2) on the role physical scale, which was below that of the general Danish population range (mean 83.1, SD 31.7). ${ }^{41}$ By comparison with the control group the intervention group showed a lesser degree of limitation by their physical status when carrying out daily activities after intervention. Overall, they are ranked at a considerably lower level than the general population. By contrast, the patients in the intervention group scored high on the physical functioning scale at baseline (mean 84.3, SD 37.2) and after the six week intervention (mean 88.2, SD 13.2), their score was similar to that of the general Danish population (mean 87.6, SD 19.4). ${ }^{41}$ This finding confirms possibly the patient group's predisposition for doing physical activity and that the intervention group was prepared to partake in demanding activities without health related constraints. With respect to the role emotional, mental health scales, and mental component scale, the patients in the intervention group showed significant improvement but had lower scores than their age equivalents in the general population.

In summary, our results in patient rated outcomes show small to medium effect sizes across a broad spectrum of physical and emotional wellbeing scales, including vitality and self reported physical activity levels. Furthermore, we found a reduction in fatigue, which we consider to be of importance to the patients' daily lives, even though no change was seen in the global health status/quality of life.

Objectively measured physiological outcomes also showed significant improvement in aerobic capacity and muscular strength. Studies in healthy adults and in people with cardiac and renal illnesses and with diabetes have shown that combined resistance and cardiovascular training programmes can have a range of beneficial effects such as increased physical function, aerobic capacity, and reduced muscular fatigue. ${ }^{42-46}$ Similarly, the results of the present study confirm that patients with cancer, even those undergoing chemotherapy, gain physiological benefits from combined resistance and cardiovascular training programmes.

According to a review of 26 studies by Galvao and Newton, the present study is the first of its kind to incorporate a high intensity design. ${ }^{47}$ The results indicate that the high intensity design is an effective training programme as both muscle strength and overall fitness improved during a short time period. Patients with evidence of disease experienced the same improvement as patients with no evidence of disease. Improvements in muscle strength and fitness in this intervention are within the same range as in the exercise interventions that included patients with lesser burden of disease post-treatment and who showed improvement in specific areas despite longer training periods. The duration and frequency of the high intensity training sessions in the present study (270 minutes per week) imply a larger training volume than in single mode exercise interventions (120-135 minutes per week). ${ }^{7947}$

Improvement in the patients' self reported vitality and physical functioning were probably attributable to the high intensity component, since a connection exists between high intensity training and vitality in healthy athletes. ${ }^{48}$

On days with high intensity exercise training, the sessions closed with low intensity relaxation training. The aim was to assist the patients to recognise and test their own physical reactions, such as dizziness, overexertion, and cold sweat. The patients were advised to concentrate on breathing and muscle tension, and gain awareness of their physical and mental reactions. In this way the patients stabilised physically and emotionally before returning to their daily activities. These findings correspond to single intervention studies regarding patients' perception of self control. ${ }^{49} \mathrm{On}$ the other hand the intervention did not affect the 
patients' levels of nausea, vomiting and pain suggesting non-effectiveness or simply that the patients in this study had a low level of symptoms at baseline.

Incorporating a heterogeneous group of cancer patients, each presenting different treatment related side effects, increases the need for daily pre-exercise screening by clinical nurse specialists. This has been of importance in preventing serious adverse events during high intensity programmes. Five participants were excluded from the high intensity physical training component (total of 25 times) during the study period because of leucopenia and/or increased blood pressure. Intermittent increase in heart rate and/or blood pressure were commonly observed in two or three patients weekly. In these cases, values for heart rate and blood pressure were retained after 10 minutes of recline, to meet pre-screening criteria and approval to enter the exercise room.

Five participants with breast cancer had lymphoedema at baseline; none experienced exacerbations during the intervention. One participant with a brain tumour experienced a grade 3 seizure after cardiovascular training. This participant was admitted to the hospital, recovered within 3 hours, and was discharged the same day. The participant was subsequently excluded from the intervention. We must therefore advise patients with brain tumours or brain metastases not to participate in high intensity exercise interventions. The inclusion and exclusion criteria, the daily screening procedures, the presence of the clinical nurse specialist during training, and training sphygmomanometer ensured the required level of safety. The combination of a training facility at the hospital and professional supervision was crucial and confirmed the patients' understanding that the training programme was a supplement to medical treatment. Despite the multimodal intervention being short term (six weeks) the volume of the intervention seemed adequate.

\begin{tabular}{|c|c|c|c|c|}
\hline & \multicolumn{2}{|c|}{ Mean (SD) } & \multicolumn{2}{|c|}{ Test (reference control) } \\
\hline & Baseline & 6 weeks & $\begin{array}{l}\text { Estimated mean } \\
\text { difference }(95 \% \mathrm{Cl})\end{array}$ & $\mathrm{P}^{*}$ \\
\hline \multicolumn{5}{|l|}{$\mathrm{Vo}_{2} \max (\mathrm{l} / \mathrm{min})$} \\
\hline Control & $1.90(0.5)$ & $1.88(0.5)$ & & \\
\hline Intervention & $1.82(0.4)$ & $1.96(0.5)$ & 0.16 (0.1 to 0.2$)$ & $<0.0001$ \\
\hline \multicolumn{5}{|c|}{ Muscular strength $(\mathrm{kg})$} \\
\hline \multicolumn{5}{|l|}{ Leg press } \\
\hline Control & $107.6(33.3)$ & $110.4(36.0)$ & & \\
\hline Intervention & $100.8(30.5)$ & $132.4(42.3)$ & 29.7 (23.4 to 34.9) & $<0.0001$ \\
\hline \multicolumn{5}{|l|}{ Chest press } \\
\hline Control & $40.2(18.0)$ & $39.7(17.2)$ & & \\
\hline Intervention & $37.9(15.6)$ & $45.2(17.9)$ & 7.5 (5.6 to 9.4$)$ & $<0.0001$ \\
\hline \multicolumn{5}{|l|}{ Pull-down } \\
\hline Control & $42.0(16.3)$ & $42.8(16.1)$ & & \\
\hline Intervention & $39.6(14.0)$ & $47.2(14.4)$ & $6.4(4.5$ to 8.3$)$ & $<0.0001$ \\
\hline
\end{tabular}

\section{Strengths and weaknesses}

The trial had a large sample size and included male and female patients with advanced cancer and different types of diagnoses. The strengths of the trial include supervised and structured exercise, combined high and low intensity components, use of validated objective physiological measurements, validated questionnaires, intention to treat analyses, and limited dropout rate of $12.7 \%$. All participants were undergoing chemotherapy during the study period. Limitations included adherence rate of $70.8 \%$ and a $53 \%$ recruitment rate, which are comparable with other exercise interventions including cancer patients with lesser disease burden. ${ }^{334}$

One weakness of the study is that while we were able to report the immediate effects of the intervention it was not possible to perform valid comparisons of the effect between the control and the intervention groups 3 months after intervention. The reason is that $59.7 \%$ of the control group patients subsequently elected to participate in the intervention following their six week participation in the study. Study allocation was not concealed, either to the patient or to the healthcare professionals, and the control group was allowed to freely increase physical activity.

Another weakness of the study is that the professionals conducting the daily exercise sessions also collected the data, which may have led to significant bias. However, research assistants who were not involved with the participants keyed in and analysed the data.

Self selection of participants in our study resulted in a sample of cancer patients who were overtly motivated to engage in group based physical activity.

Our findings suggest that population heterogeneity does not preclude use of this type of intervention. However, generalisation may be limited by the willingness of patients to allocate the necessary time to physical activity. Furthermore, all patients in the present study had a good performance status (WHO 0-1) and did not have brain or bone metastases. Our intervention may need to be modified in patients with a performance status of 2 or greater. Finally, we consider it a great challenge to motivate patients who are not interested in physical training in groups with mixed gender.

\section{Meaning}

There is a considerable rationale for promoting multimodal exercise interventions to improve physical capacity, vitality, and physical and mental wellbeing, and to relieve fatigue during chemotherapy; thereby supporting cancer patients' daily living activities. The range of exercise components used, from high intensity activity to relaxation, body awareness training, and massage has been shown to be feasible, safe, and beneficial to various patients with cancer during chemotherapy, even patients with advanced disease.

Very few exercise interventions include cancer patients with advanced disease and most of these involve patients with haematological disease during 


\section{WHAT IS ALREADY KNOWN ON THIS TOPIC}

Supervised cardiovascular exercise training of moderate intensity has shown benefits in patients with breast cancer during or after adjuvant chemotherapy

Supervised group exercise interventions including patients with different cancer diagnoses and with advanced disease undergoing chemotherapy have not been evaluated in randomised clinical trials

\section{WHAT THIS PAPER ADDS}

High intensity exercise can be undertaken safely by such patients and is associated with physiological, functional, and emotional benefits

Multimodal high and low intensity exercise interventions can be offered by interdisciplinary teams of clinicians

Innovative approaches to address men's needs for physical rehabilitation in this context are required
8 McNeely ML, Campbell KL, Rowe BH, Klassen TP, Mackey JR, Courneya KS. Effects of exercise on breast cancer patients and survivors: a systematic review and meta-analysis. CMAJ 2006;175:34-41.

9 Markes M, Brockow T, Resch KL. Exercise for women receiving adjuvant therapy for breast cancer. Cochrane Database Syst Rev 2006;(4):CD005001.

10 Kraemer WJ, Adams K, Cafarelli E, Dudley GA, Dooly C, Feigenbaum MS, et al. American College of Sports Medicine position stand: progression models in resistance training for healthy adults. Med Sci Sports Exerc 2002;34:364-80.

11 Kangas M, Bovbjerg DH, Montgomery GH. Cancer-related fatigue: a systematic and meta-analytic review of non-pharmacological therapies for cancer patients. Psychol Bull 2008;134:700-41.

12 Kwekkeboom KL, Hau H, Wanta B, Bumpus M. Patients' perceptions of the effectiveness of guided imagery and progressive muscle relaxation interventions used for cancer pain. Complement Ther Clin Pract 2008;14:185-94

13 Ainsworth BE, Haskell WL, Whitt MC, Irwin ML, Swartz AM, Strath S, et al. Compendium of physical activities: an update of activity codes and MET intensities. Med Sci Sports Exerc 2000;32(9 Suppl):S498-504.

14 Saltin B, Gollnick PD. Skeletal muscle adaptability: significance for metabolism and performance. In: Peachey LD, Adrian PH, Geiger SR, eds. Handbook of physiology, sektion 10: skeletal muscle. American Physiological Society, 1983:555-631.

or after high dose chemotherapy and peripheral blood stem cell transplantation. ${ }^{5051}$ Patients with advanced solid cancers differ substantially from these patientsthus investigations including such patients are still needed. The present study had a clear majority of female patients $(73 \%)$. However, the number of people with cancer each year is evenly distributed between the two sexes. ${ }^{52-56}$ We therefore recommend that interventions should be developed with greater appeal to male patients.

Contributors: LA, CA, MQ, TM, JM, and MR contributed to conception and trial design. LA, JM, MR, and JH wrote the protocol. JH, KV, BC, CA, and TM were responsible for recruitment of patients to the trial. $M Q, C A$, and $T M$ conducted the daily training sessions. CA, MQ, TM, and BC collected the data. JH, MR, and KV provided clinical expertise. DK was study statistician. MTB, MS and MK contributed to the statistical analysis and interpretation of data. LA and MR drafted the first version of the manuscript. LA obtained the funding. JH, DK, KV, MQ TM, CA, BC, and JM critically reviewed, revised, and supplemented the manuscript. All authors approved the final version. LA is the guarantor.

Funding: This research was supported by grants from The Lundbeck Foundation, The Novo Nordic Foundation, The Egmont Foundation, The Danish Cancer Society, The Svend Andersen Foundation, The Aase and Ejnar Danielsen Foundation, The Beckett Foundation, The WedellWedellsborg Foundation, The Hede Nielsen Family Foundation, The Gangsted Foundation, Copenhagen University Hospital. The authors' work was independent from the funders.

Competing interests: None declared.

Ethical approval: Ethical approval was obtained from the Scientific Committees of the Copenhagen and Frederiksberg Municipalities (j.no. 01273/00) and the Danish Data Protection Agency (j.no. 2000-41-0149).

1 Smets EM, Garssen B, Schuster-Uitterhoeve AL, de Haes JC. Fatigue in cancer patients. Br / Cancer 1993;68:220-4.

2 Henry DH, Viswanathan HN, Elkin EP, Traina S, Wade S, Cella D. Symptoms and treatment burden associated with cancer treatment: results from a cross-sectional national survey in the US. Support Care Cancer 2008;16:791-801.

3 Baum A, Andersen BL. Introduction. In: Baum A, Andersen BL, eds. Psychosocial interventions for cancer. American Psychological Association, 2001:3-15.

4 Pinto BM, Eakin E, Maruyama NC. Health behavior changes after a cancer diagnosis: what do we know and where do we go from here? Ann Behav Med 2000;22:38-52.

5 Fentem PH. ABC of sports medicine: benefits of exercise in health and disease. BMJ 1994;308:1291-5.

6 Lucía A, Earnest C, Pérez M. Cancer-related fatigue: can exercise physiology assist oncologists? Lancet Oncol 2003;4:616-25.

7 Schmitz KH, Holtzman J, Courneya KS, Mâsse LC, Duval S, Kane R. Controlled physical activity trials in cancer survivors: a systematic review and meta-analysis. Cancer Epidemiol Biomarkers Prev 2005;14:1588-95.
15 McCaffery M, Beebe A. Pain: clinical manual for nursing practice. Mosby, 1989.

16 da Costa BR, Vieira ER. Stretching to reduce work-related musculoskeletal disorders: a systematic review. J Rehabil Med 2008;40:321-8

17 Bower JE, Woolery A, Sternlieb B, Garet D. Yoga for cancer patients and survivors. Cancer Control 2005;12:165-71.

18 Anderson BD, Spector AH. Introduction to pilates-based rehabilitation. Orthop Phys Ther Clin N Am 2000;9:395-400.

19 Ferrell-Torry AT, Glick OJ. The use of therapeutic massage as a nursing intervention to modify anxiety and the perception of cancer pain. Cancer Nurs 1993;16:93-101.

20 Aaronson NK, Ahmedzai S, Bergman B, Bullinger M, Cull A, Duez NJ, et al. The European Organization for Research and Treatment of Cancer QLQ-C30: a quality-of-life instrument for use in international clinical trials in oncology. J Natl Cancer Inst 1993;85:365-76.

21 Ware JE Jr, Sherbourne CD. The MOS 36-item short-form health survey (SF-36). I. Conceptual framework and item selection. Med Care

22 Saltin B, Grimby G. Physiological analysis of middle-aged and old former athletes. Comparison with still active athletes of the same ages. Circulation 1968;38:1104-15.

23 de Bruin A, Picavet HSJ, Nossikov A, eds. Health interview surveys: towards international harmonization of methods and instruments. World Health Organization, 1996.

24 Aadahl M, Jørgensen T. Validation of a new self-report instrument for measuring physical activity. Med Sci Sports Exerc 2003;35:1196-202.

25 Brzycki M. Strength testing: predicting a one-rep max from reps to fatigue. J Phys Educ Recreation Dance 1993;64:88-90.

26 Andersen LB. A maximal cycle exercise protocol to predict maximal oxygen uptake. Scand J Med Sci Sports 1995;5:143-6.

27 Quist M, Rorth M, Zacho M, Andersen C, Moeller T, Midtgaard J, Adamsen L. High-intensity resistance and cardiovascular training improve physical capacity in cancer patients undergoing chemotherapy. Scand I Med Sci Sports 2006;16:349-57.

28 Adamsen L, Quist M, Midtgaard J, Andersen C, Møller T, Knutsen L et al. The effect of a multidimensional exercise intervention on physical capacity, well-being and quality of life in cancer patients undergoing chemotherapy. Support Care Cancer 2006;14:116-27.

29 Cohen J. Statistical power analysis for the behavioral sciences. 2nd rev ed. Lawrence Erlbaum Associates, 1988.

30 Nakagawa S, Cuthill IC. Effect size, confidence interval and statistical significance: a practical guide for biologists. Biol Rev Camb Philos Soc 2007;82:591-605.

31 Klee M, Groenvold M, Machin D. Quality of life of Danish women: population-based norms of the EORTC QLQ-C30. Qual Life Res 1997;6:27-34

32 King MT. The interpretation of scores from the EORTC quality of life questionnaire QLQ-C30. Qual Life Res 1996;5:555-67.

33 Mutrie N, Campbell AM, Whyte F, McConnachie A, Emslie C, Lee L, et al. Benefits of supervised group exercise programme for women being treated for early stage breast cancer: pragmatic randomised controlled trial. $B M$ I 2007;334:517-20. Friedenreich CM, et al. Effects of aerobic and resistance exercise in breast cancer patients receiving adjuvant chemotherapy: a multicenter randomized controlled trial. J Clin Oncol 1992;30:473-83.

34 Courneya KS, Segal RJ, Mackey JR, Gelmon K, Reid RD, 2007;25:4396-404. 
35 Lucía A, Earnest C, Pérez M. Cancer-related fatigue: can exercise physiology assist oncologists? Lancet Oncol 2003;4:616-25.

36 Adamsen L, Midtgaard J, Roerth M, Andersen C, Quist M, Moeller T. Transforming the nature of fatigue through exercise: qualitative findings from a multidimensional exercise programme in cancer patients undergoing chemotherapy. Eur / Cancer Care 2004;13:362-70.

37 Uitterhoeve RJ, Vernooy M, Litjens M, Potting K, Bensing J, De Mulder $\mathrm{P}$, et al. Psychosocial interventions for patients with advanced cancer: a systematic review of the literature. Br J Cancer 2004;91:1050-62.

38 van Emmerik AA, Kamphuis JH, Hulsbosch AM, Emmelkamp PM. Single session debriefing after psychological trauma: a metaanalysis. Lancet 2002;360:766-71.

39 Roila F, Hesketh PJ, Herrstedt J; Antiemetic Subcommittee of the Multinational Association of Supportive Care in Cancer. Prevention of chemotherapy- and radiotherapy-induced emesis: results of the 2004 Perugia International Antiemetic Consensus Conference. Ann Oncol 2006;17:20-8.

40 Segal R, Evans W, Johnson D, Smith J, Colletta S, Gayton J, et al. Structured exercise improves physical functioning in women with stages I and II breast cancer: results of a randomized controlled trial. Clin Oncol 2001;19:657-65

41 Bjørner JB, Damsgaard MT, Watt T, Bech P, Rasmussen NK, Kristensen TS, et al. Dansk manual til SF-36: et spørgeskema om helbredsstatus [SF-36 (Danish manual): health-related quality of life questionnaire]. Danish Association of the Pharmaceutical Industry, 1997.

42 Nader GA. Concurrent strength and endurance training: from molecules to man. Med Sci Sports Exerc 2006;38:1965-70.

43 Gayda M, Choquet D, Ahmaidi S. Effects of exercise training modality on skeletal muscle fatigue in men with coronary heart disease. J Electromyogr Kinesiol 2009;19:e32-9.

44 Moinuddin I, Leehey DJ. A comparison of aerobic exercise and resistance training in patients with and without chronic kidney disease. Adv Chronic Kidney Dis 2008;15:83-96.

45 Thomas DE, Elliott EJ, Naughton GA. Exercise for type 2 diabetes mellitus. Cochrane Database Syst Rev 2006;(3):CD002968.
46 Milne HM, Wallman KE, Gordon S, Courneya KS. Effects of a combined aerobic and resistance exercise program in breast cancer survivors: a randomized controlled trial. Breast Cancer Res Treat 2008;108:279-88.

47 Galvão DA, Newton RU. Review of exercise intervention studies in cancer patients. J Clin Oncol 2005;23:899-909.

48 O'Connor PJ, Puetz TW. Chronic physical activity and feelings of energy and fatigue. Med Sci Sports Exerc 2005;37:299-305.

49 Luebbert K, Dahme B, Hasenbring M. The effectiveness of relaxation training in reducing treatment-related symptoms and improving emotional adjustment in acute non-surgical cancer treatment: a meta-analytical review. Psychooncology 2001;10:490-502.

50 Dimeo F, Schwartz S, Fietz T, Wanjura T, Böning D, Thiel E. Effects of endurance training on the physical performance of patients with hematological malignancies during chemotherapy. Support Care Cancer 2003;11:623-8.

51 Jarden M, Hovgaard D, Boesen E, Quist M, Adamsen L. Pilot study of a multimodal intervention: mixed-type exercise and psychoeducation in patients undergoing allogeneic stem cell transplantation. Bone Marrow Transplant 2007;40:793-800.

52 Cancer Research UK. UK cancer incidence statistics by country: table 8.2: females—number of new cases of cancer diagnosed, by site, UK, 2005. http://publications.cancerresearchuk.org/WebRoot/ crukstoredb/CRUK_PDFs/incidence/cs_inc_t8.2.pdf.

53 Cancer Research UK. UK cancer incidence statistics by country: table 8.1: males-number of new cases of cancer diagnosed, by site, UK, 2005. http://publications.cancerresearchuk.org/WebRoot/ crukstoredb/CRUK_PDFs/incidence/cs_inc_t8.1.pdf.

54 Espey DK, Wu XC, Swan J, Wiggins C, Jim MA, Ward E, et al. Annual report to the nation on the status of cancer, 1975-2004, featuring cancer in American Indians and Alaska Natives. Cancer 2007;110:2119-52.

55 Canadian Cancer Society. Canadian cancer statistics 2008. Toronto: National Cancer Institute of Canada, 2008.

56 Clemmensen IH, Nedergaard KH, Storm HH. Kræft i Danmark: en opslagsbog[Prevalence of cancer in Denmark: a reference book]. Danish Cancer Society, 2006.

Accepted: 8 May 2009 